\title{
Entropic uncertainty and the quantum phase transition in the Dicke model
}

\author{
E. Romera \\ Departamento de Física Atómica, Molecular y Nuclear and Instituto Carlos I de Física Teórica y Computacional, \\ Universidad de Granada, Fuentenueva s/n, 18071 Granada, Spain \\ M. Calixto \\ Departamento de Matemática Aplicada, Universidad de Granada, Fuentenueva s/n, 18071 Granada, Spain
}

\author{
Á. Nagy \\ Department of Theoretical Physics, University of Debrecen, H-4010 Debrecen, Hungary
}

(Dated: November 26, 2011)

\begin{abstract}
We show that the description of the quantum phase transition in terms of the entropic uncertainty relation turns out to be more suitable than in terms of the standard variance-based uncertainty relation. The entropic uncertainty relation detects the quantum phase transition in the Dicke model and it provides a correct description of the quantum fluctuations or quantum uncertainty of the system.
\end{abstract}

Classical phase transitions describe an abrupt modification in the physical properties of a system due to the change of a parameter (normally the temperature), and the phenomena responsible for them are classical fluctuations (thermal fluctuations in case the temperature was the parameter). An important extension of this concept is to quantum systems at zero temperature where there is an abrupt change in the physical properties induced by the modification of certain couplings $\lambda$ which describe the interaction between the microscopic constituents of the system (the Hamiltonian is $H=H(\lambda)=H_{0}+\lambda V$ with $H_{0}$ integrable) and where the so-called quantum fluctuations are responsible for a dramatic change in the properties of the system. At the critical point $\lambda=\lambda_{c}$, there is an abrupt change in the symmetry of the ground-state wavefunction [?].

Quantum phase transitions (QPT) are induced by quantum uncertainty. The Heisenberg uncertainty principle can be expressed in terms of the familiar variancebased uncertainty relation. Entropic uncertainty relation [? ? ? ] provides an alternative to express quantitatively the uncertainty principle in several situations [? ? ? ? ? ? ? ? ].

In this letter we characterize quantum fluctuations in terms of the entropic uncertainty relation in a quantum phase transition. We show that the entropic uncertainty relation [? ] gives a more appropriate description of the QPT than the usual variance-based uncertainty relation in the Dicke model, providing an excellent marker of QPT.

We shall consider the Dicke model that describes a single-mode bosonic field interacting with an ensemble of $N$ two-level atoms. There is a QPT in the $N \rightarrow \infty$ limit. This model is of great importance in quantum optics [? ? ? ? ? ]. It has also been used to relate QPT with chaotic [? ? ] or entanglement [? ] properties. The Dicke model has been realized with a superfluid gas in an optical cavity [? ] and the spontaneous symmetry breaking has been observed recently [? ].

The single-mode Dicke Hamiltonian has the form

$$
H=\omega_{0} J_{z}+\omega a^{\dagger} a+\frac{\lambda}{\sqrt{2 j}}\left(a^{\dagger}+a\right)\left(J_{+}+J_{-}\right),
$$

where $J_{z}, J_{ \pm}$are the angular momentum operators for a pseudospin of length $j=N / 2$. The ensemble of two-level atoms with level-splitting $\omega_{0}$ are considered. $a$ and $a^{\dagger}$ are the bosonic operators of the field (the bosonic mode has a frequency $\omega$ ). In the thermodynamic limit, where the number of atoms becomes infinite $(N, j \rightarrow \infty)$, there is a QPT at a critical value of the atom-field coupling strength $\lambda_{c}=\frac{1}{2} \sqrt{\omega \omega_{0}}$. There are two phases: normal phase $\left(\lambda<\lambda_{c}\right)$ and superradiant phase $\left(\lambda>\lambda_{c}\right)$.

Let us consider the wave functions of the Dicke Hamiltonian at finite $j$ in position representation [? ]:

$$
\begin{aligned}
\psi(x, y)= & \frac{\sqrt{\omega \omega_{0}}}{\sqrt{\pi}} e^{-1 / 2\left(\omega x^{2}+\omega_{0} y^{2}\right)} \sum_{n=0}^{n_{c}} \sum_{m=-j}^{j} c_{n m}^{(j)} \\
& \times \frac{H_{n}(\sqrt{\omega} x) H_{j+m}\left(\sqrt{\omega_{0}} y\right)}{2^{(n+m+j) / 2} \sqrt{n !(j+m) !}}
\end{aligned}
$$

where $n_{c}$ is the dimension of the truncated bosonic Hilbert space and $H_{n}$ are the Hermite polynomials. The coefficients $c_{n m}^{(j)}$ are determined by numerical diagonalization which is carried out using the basis $\{|n\rangle \otimes|j, m\rangle\}$ with $|n\rangle$ the number states of the field and $|j, m\rangle$ the Dicke states, where the position representation of the number states $|n\rangle$ and $|j, m\rangle$ are given by $\langle x \mid n\rangle=$ $e^{-(1 / 2) \omega x^{2}} H_{n}(\sqrt{\omega} x) \omega /\left(\sqrt{2^{n} n ! \sqrt{\pi}}\right)$ and $\langle y \mid j, m\rangle=$ $e^{-(1 / 2) \omega_{0} y^{2}} H_{j+m}\left(\sqrt{\omega_{0}} y\right) \omega_{0} /\left(\sqrt{\left.2^{(} j+m\right)(j+m) ! \sqrt{\pi}}\right)$,

respectively (see [?] for details). The momentum space wavepacket $\phi\left(p_{x}, p_{y}\right)$ is the Fourier transform of $\psi(x, y)$.

We shall denote the probability densities in position and momentum spaces as $\rho(x, y)=|\psi(x, y)|^{2}$ and 


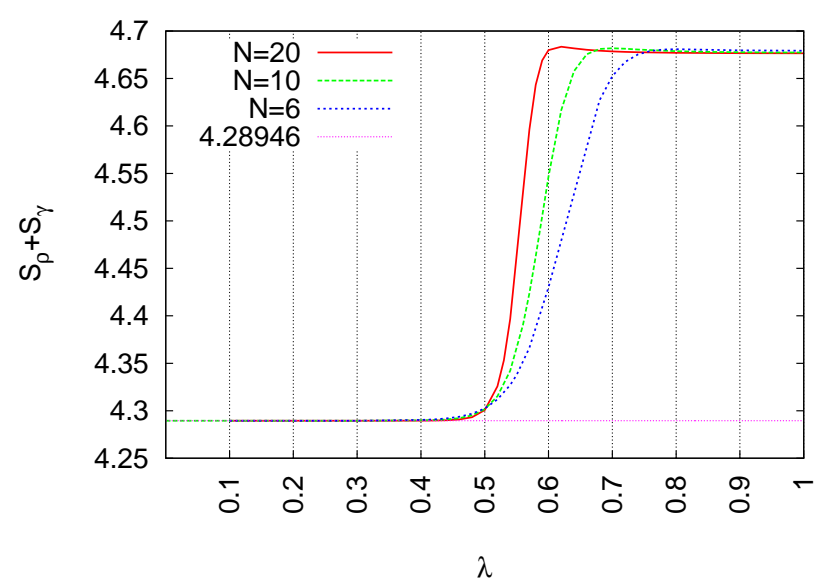

FIG. 1: Shannon entropy sum $S_{\rho}+S_{\gamma}$ for the ground state for $N=6,10$ and 20 and $\omega_{0}=\omega=1$. Note the value $2(1+\ln \pi) \approx 4.29$

$\gamma\left(p_{x}, p_{y}\right)=\left|\phi\left(p_{x}, p_{y}\right)\right|^{2}$, respectively. The uncertainty relation for the Shannon information entropy [? ] has the form

$$
S_{\rho}+S_{\gamma} \geq 2(1+\ln \pi)
$$

where $S_{f} \equiv-\int f(\mathbf{r}) \ln f(\mathbf{r}) d \mathbf{r}$.

The variance-based Heisenberg uncertainty relation, on the other hand, is given by

$$
\Delta x \Delta p_{x} \geq 1 / 2 \text { and } \Delta y \Delta p_{y} \geq 1 / 2 .
$$

Figs. ?? and ?? present the Shannon entropy sum $S_{\rho}+$ $S_{\gamma}(? ?)$ and the variance products $\Delta x \Delta p_{x}$ and $\Delta y \Delta p_{y}$ (??), respectively, as a function of $\lambda$ for different values of $N$. In all calculations we have taken $\omega_{0}=\omega=1$, that is, the Hamiltonian is in a scaled resonance, so $\lambda_{c}=$ 0.5 . For values of $\lambda<\lambda_{c}$, the Shannon entropy sum has the value $2(1+\ln \pi)$ and the variance products are $1 / 2$, approximately. As $\lambda$ increases for $\lambda>\lambda_{c}$, the entropy sum goes to 4.68 with a step discontinuity as $N$ increases. However, for any value of $\lambda$ with $\lambda>\lambda_{c}$, the variance uncertainty products diverge when $N$ goes to infinity.

The ground-state density function (??) is presented for different values of $\lambda$ in Fig. ??. In the normal phase it is a Gaussian-like packet centered at the origin and, as the strength of the coupling goes around $\lambda_{c}$, it splits into two overlapping subpackets. When the coupling is greater than $\lambda_{c}$ these two overlapping subpackets move away from each other into different quadrants in the plane. This behavior is displayed in Fig. ?? for $N=20$ and (from top to bottom) for $\lambda=0.3, \lambda=0.55$ and $\lambda=0.7$ (it has also been previously studied in Ref.[? ]). One can see that the appearance of two different subpackets is captured by entropic uncertainty relation in (Fig. ??) where we have a constant finite value when $N$ goes to infinity and $\lambda>\lambda_{c}$. Clearly, this information isn't provided by the variance-based uncertainty relation (in Fig.

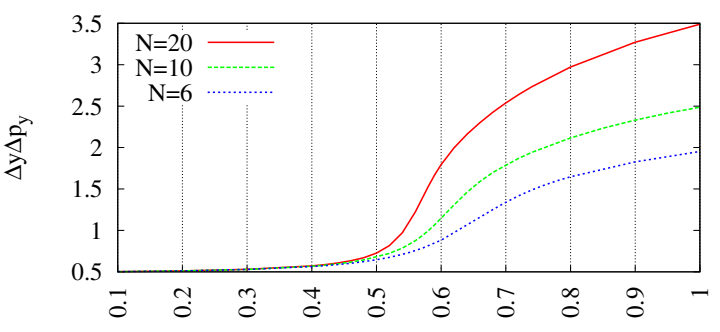

$\lambda$

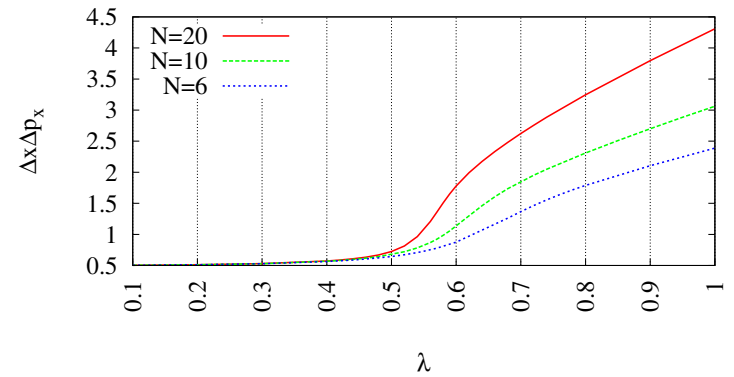

FIG. 2: Variance products $\Delta x \Delta p_{x}$ and $\Delta y \Delta p_{y}$ for the ground state for $N=6,10$ and 20 and $\omega_{0}=\omega=1$.
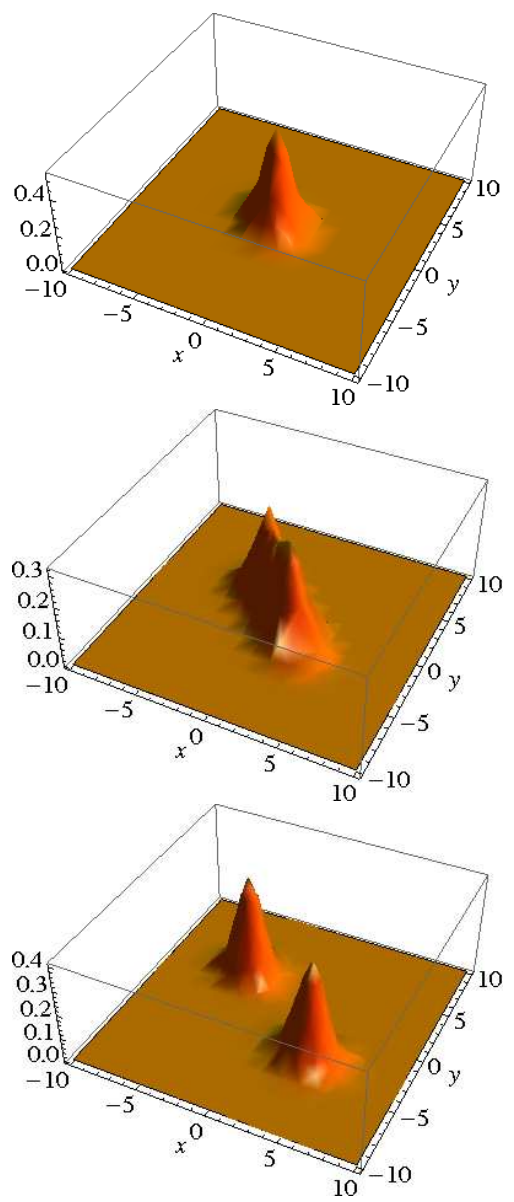

FIG. 3: Ground state density function for different values of $\lambda$ (from top to bottom $\lambda=0.3, \lambda=0.55$ and $\lambda=0.7$ ) for $\omega_{0}=\omega=1$ and $N=20$. 
??) which has a divergence when $N$ goes to infinity. This important difference is due to the fact that the entropic uncertainty relation considers the quantum fluctuations or uncertainty of the subpackets independently of their relative position, whereas the variance-based uncertainty relation takes into account not only the quantum fluctuations properly, but also the relative position between the subpackets.

Now we present analytical expressions for uncertainty relations using trial states expressed in terms of "symmetry-adapted coherent states" introduced by Castaños et al. [? ], which turn out to be an excellent approximation to the exact quantum solution of the ground $(+)$ and first excited $(-)$ states of the Dicke model. These variational states are obtained by applying projectors of even and odd parity to the direct product $|\alpha\rangle \otimes|z\rangle$, to obtain

$$
|\alpha, z\rangle_{ \pm}=\mathcal{N}_{ \pm}(|\alpha\rangle \otimes|z\rangle \pm|-\alpha\rangle \otimes|-z\rangle),
$$

where $\mathcal{N}_{ \pm}$is a normalization factor and

$$
\begin{aligned}
& |\alpha\rangle=e^{-|\alpha|^{2} / 2} e^{\alpha a^{\dagger}}|0\rangle=e^{-|\alpha|^{2} / 2} \sum_{n=0}^{\infty} \frac{\alpha^{n}}{\sqrt{n !}}|n\rangle, \\
& |z\rangle=\left(1+|z|^{2}\right)^{-j} e^{z J_{+}}|j,-j\rangle= \\
& \left(1+|z|^{2}\right)^{-j} \sum_{m=-j}^{j}\left(\begin{array}{c}
2 j \\
j+m
\end{array}\right)^{1 / 2} z^{j+m}|j, m\rangle,
\end{aligned}
$$

are the ordinary (standard) and spin- $j$ coherent states for the photon and the particle sectors, respectively. The critical points

$$
\begin{aligned}
& \alpha=\alpha_{0}= \begin{cases}0, & \text { if } \lambda<\lambda_{c} \\
-\sqrt{2 j} \sqrt{\frac{\omega_{0}}{\omega}} \frac{\lambda}{\lambda_{c}} \sqrt{1-\left(\frac{\lambda}{\lambda_{c}}\right)^{-4}}, & \text { if } \lambda \geq \lambda_{c}\end{cases} \\
& z=z_{0}= \begin{cases}0, & \text { if } \lambda<\lambda_{c} \\
\sqrt{\frac{\frac{\lambda}{\lambda_{c}}-\left(\frac{\lambda}{\lambda_{c}}\right)^{-1}}{\lambda_{c}}+\left(\frac{\lambda}{\lambda_{c}}\right)^{-1}} & \text { if } \lambda \geq \lambda_{c}\end{cases}
\end{aligned}
$$

minimize the mean energy $\langle H\rangle_{+} \equiv_{+}\langle\alpha, z|H| \alpha, z\rangle_{+}$, except in a close neighborhood around $\lambda_{c}$ which diminishes as $j$ increases (see [?]).

In order to compute uncertainty relations for information entropies in position and momentum representations, we shall make use of the Holstein-Primakoff representation [? ] of the angular momentum operators $J_{ \pm}$ in terms of the bosonic operators, $\left[b, b^{\dagger}\right]=1$, given by:

$$
\begin{gathered}
J_{+}=b^{\dagger} \sqrt{2 j-b^{\dagger} b} \simeq \sqrt{2 j}, \quad J_{-}=\sqrt{2 j-b^{\dagger} b} b \\
J_{z}=\left(b^{\dagger} b-j\right) .
\end{gathered}
$$

Redefining $\beta \equiv \sqrt{2 j} z$, it can be seen (see e.g. [? ? ]) that spin- $j$ coherent states $|z\rangle$ go over to ordinary coherent states $|\beta\rangle \equiv e^{|\beta|^{2} / 2} e^{\beta b^{\dagger}}|0\rangle$ for $j>>1$ (when identifying $|j,-j\rangle \equiv|0\rangle)$. Thus, we shall assume the approximation:

$$
|z\rangle \simeq|\beta\rangle,
$$

which turns out to be a quite good estimate even for relatively small values of $j$. Introducing position and momentum operators for the two bosonic modes as usual:

$$
\begin{aligned}
& X=\frac{1}{\sqrt{2 \omega}}\left(a^{\dagger}+a\right), \quad P_{X}=i \sqrt{\frac{\omega}{2}}\left(a^{\dagger}-a\right), \\
& Y=\frac{1}{\sqrt{2 \omega}}\left(b^{\dagger}+b\right), \quad P_{Y}=i \sqrt{\frac{\omega_{0}}{2}}\left(b^{\dagger}+b\right),
\end{aligned}
$$

the explicit expression of the ground state wave function $\left|\alpha_{0}, \beta_{0}\right\rangle_{+}$in position $\left(\psi(x, y)=\left\langle x, y \mid \alpha_{0}, \beta_{0}\right\rangle_{+}\right)$and momentum $\left(\phi\left(p_{x}, p_{y}\right)=\left\langle p_{x}, p_{y} \mid \alpha_{0}, \beta_{0}\right\rangle_{+}\right)$representations can be easily obtained as (see e.g. [? ]):

$$
\begin{aligned}
& \psi(x, y)=\mathcal{N}_{+}\left(e^{-\frac{\left(\sqrt{\omega} x-\sqrt{2} \alpha_{0}\right)^{2}}{2}-\frac{\left(\sqrt{\omega_{0}} y-\sqrt{2} \beta_{0}\right)^{2}}{2}}\right. \\
& \left.+e^{-\frac{\left(\sqrt{\omega} x+\sqrt{2} \alpha_{0}\right)^{2}}{2}-\frac{\left(\sqrt{\omega} y+\sqrt{2} \beta_{0}\right)^{2}}{2}}\right), \\
& \phi\left(p_{x}, p_{y}\right)=2 \mathcal{N}_{+} e^{-\frac{p_{x}^{2}}{2 \omega}-\frac{p_{y}^{2}}{2 \omega_{0}}} \cos \left(\sqrt{2}\left(\frac{p_{x}}{\sqrt{\omega}} \alpha_{0}+\frac{p_{y}}{\sqrt{\omega_{0}}} \beta_{0}\right)\right),
\end{aligned}
$$

where $\mathcal{N}_{+}=\left(\frac{2 \pi}{\omega_{0} \omega}\left(1+e^{-2 \alpha_{0}^{2}-2 \beta_{0}^{2}}\right)\right)^{-1 / 2}$ is a normalization factor. Note that for $\lambda>\lambda_{c}$ the ground-state density function $\rho(x, y)=|\psi(x, y)|^{2}$ splits up into two Gaussian packets centered at antipodal points in the $x-y$ plane. The packets move away from each other for increasing $j$. In momentum space, $\gamma\left(p_{x}, p_{y}\right)=\left|\phi\left(p_{x}, p_{y}\right)\right|^{2}$ is a Gaussian modulated by a cosine function which oscillates rapidly for high $j$ for $\lambda>\lambda_{c}$. This leads to a Heaviside (step) function behavior of

$$
S_{\rho}+S_{\gamma}=\left\{\begin{array}{l}
S^{\text {normal }}=\ln (e \pi)^{2} \simeq 4.29, \quad \text { if } \lambda<\lambda_{c} \\
S^{\text {super }}=\ln \left((2 \pi)^{2} e\right) \simeq 4.68, \text { if } \lambda \geq \lambda_{c}
\end{array}\right.
$$

in the thermodynamic limit $(j \rightarrow \infty)$. This behavior can be inferred from Figures ?? and ?? panel (a). In the normal phase, the inequality (??) saturates (that is, the total entropy is exactly $\left.\ln (e \pi)^{2}\right)$ because the ground state wave function (??) is a Gaussian in position and momentum representation. Note that the rise height of the step from normal to super-radiant is $S^{\text {super }}-S^{\text {normal }}=$ $\ln (4 / e)$. We can decompose $S^{\text {super }}=S_{\rho}^{\text {super }}+S_{\gamma}^{\text {super }}$, with $S_{\rho}^{\text {super }}=S_{\rho}^{\text {normal }}+\ln (2)$ (which can be analytically calculated taking into account that the wavepacket in position representation splits into two subpackets with negligible overlap for $\left.\lambda>\lambda_{c}\right)$ and $S_{\gamma}^{\text {super }}=S_{\gamma}^{\text {normal }}+\ln (2 / e)$.

We would also like to point out that the Heaviside (step) function behavior of $S_{\rho}+S_{\gamma}$ should also appear in other quantum systems where a single wavepacket splits up into several subpackets above a critical value $\lambda_{c}$ of some parameter $\lambda$ of the theory. In particular, for $M$ identical subpackets with negligible overlap, one can see that the entropy in position representation $S_{\rho}$ increases by an amount of $\ln (M)$.

We can also compute explicit expressions for expectation values

$$
\begin{aligned}
& \langle a\rangle_{+}=\langle b\rangle_{+}=\left\langle a^{\dagger}\right\rangle_{+}=\left\langle b^{\dagger}\right\rangle_{+}=0, \\
& \left\langle a^{\dagger} a\right\rangle_{+}=\alpha_{0}^{2}\left(4 \pi \mathcal{N}_{+}^{2}-1\right), \\
& \left\langle b^{\dagger} b\right\rangle_{+}=\beta_{0}^{2}\left(4 \pi \mathcal{N}_{+}^{2}-1\right),
\end{aligned}
$$


(a)

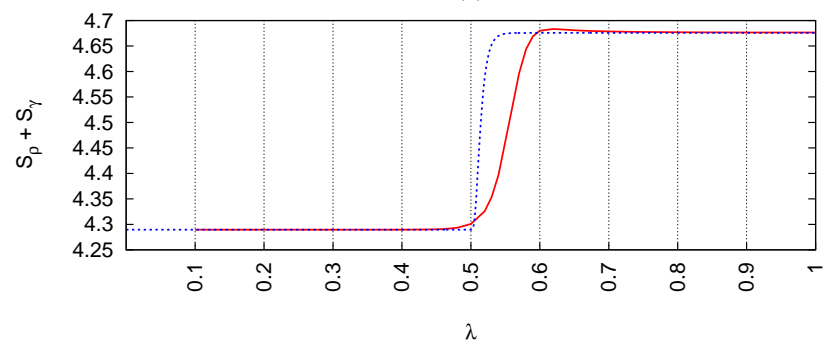

(b)

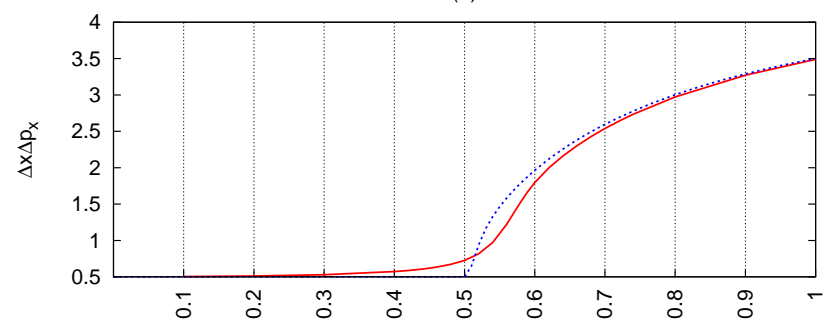

(c)

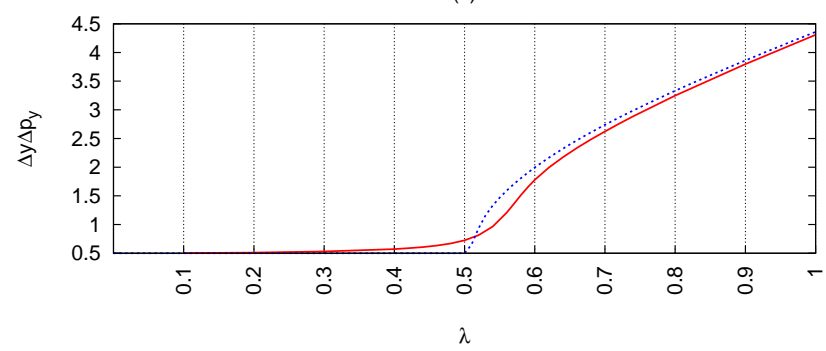

FIG. 4: Analytical approximation (dashed line) and numerical results (solid line) for (a) Shannon entropy sum $S_{\rho}+S_{\gamma}$, (b) variance products $\Delta x \Delta p_{x}$, and (c) variance products $\Delta y \Delta p_{y}$ for the ground-state for $j=10$ and $\omega_{0}=\omega=1$ $\left(\lambda_{c}=0.5\right)$.

and fluctuations

$$
\begin{aligned}
& \Delta x=\sqrt{\frac{4 \pi \mathcal{N}_{+}^{2} \alpha_{0}^{2}+\frac{1}{2}}{\omega}}, \frac{\Delta p_{x}}{\sqrt{\omega}}=\sqrt{2 \alpha_{0}^{2}\left(2 \pi \mathcal{N}_{+}^{2}-1\right)+\frac{1}{2}} \\
& \Delta y=\sqrt{\frac{4 \pi \mathcal{N}_{+}^{2} \beta_{0}^{2}+\frac{1}{2}}{\omega_{0}}}, \quad \frac{\Delta p_{y}}{\sqrt{\omega_{0}}}=\sqrt{2 \beta_{0}^{2}\left(2 \pi \mathcal{N}_{+}^{2}-1\right)+\frac{1}{2}}
\end{aligned}
$$

These analytical expressions are in agreement with our numerical calculations, as shown in Figures ?? (panel (b)) and ?? (panel(c)) .

Summarizing, the Shannon entropy sum can be considered a measure of fluctuations. In this letter we demonstrate that it remains constant even in the superradiant phase. The variance products are divergent in the superradiant phase, therefore entropic uncertainty relation provides a description of better quality.

\section{Acknowledgments}

This work was supported by the Projects: PYR-2010-
24 (Proyecto GENIL ref. CEB09-0010), FIS2008-01143 and FIS2008-06078-C03-01 (Spanish MICINN), 08814/PI/08 (Fundación Séneca), FQM-165/0207 and FQM219 (Junta de Andalucía). The work is also supported by the TAMOP 4.2.1/B-09/1/KONV-2010-0007 project. The project is cofinanced by the European Union and the European Social Fund. Grant OTKA No. K 67923 is also gratefully acknowledged.

[1] S. Sachdev, Quantum Phase Transitions, Cambridge University Press (2000).

[2] I. I. Hirschman, Am. J. Math. 79, 152 (1957)

[3] Bialynicki-Birula, I., Mycielski, J.: Commun. Math. Phys. 44, 129 (1975)

[4] I. Bialynicki-Birula, Phys. Lett. A 103, 253 (1984)

[5] H. Maassen, J. B. M. Uffink, Phys. Rev. Lett. 60, 1103 (1988)

[6] J. Sánchez-Ruiz, Phys. Lett. A 244, 189 (1998)

[7] I. Bialynicki-Birula, Phys. Rev. A 74, 052101 (2006)

[8] E. Romera, F. de los Santos Phys. Rev. Lett.99, 263601 (2007); Phys Rev. A 78, 013837 (2008).

[9] T. Schürmann and I. Hoffmann Found Phys 39, 958 (2009).

[10] W. Beckner, Ann. Math. 102, 159 (1975)

[11] S. R. Gadre, Phys. Rev. A 30, 620 (1984).

[12] S. R. Gadre, S. B. Sears, S. J. Chakrovarty and R. D. Bendale Phys. Rev. A 32, 2602 (1985).

[13] R. H. Dicke, Phys. Rev. 93, 99 (1954).

[14] K. Hepp and E. H. Lieb, Phys. Rev. A 8, 2517 (1973).

[15] Y. K. Wang and F. T. Hioe, Phys. Rev. A 7, 831 (1973)

[16] H. J. Carmichael, C. W. Gardiner, and D. F. Walls, Phys. Lett. A 46, 47 (1973).

[17] C. Emary and T. Brandes, Phys. Rev. E 67, 066203 (2003).

[18] C. Emary and T. Brandes, Phys. Rev. Lett. 90, 044101 (2003).

[19] N. Lambert, C. Emary, and T. Brandes, Phys. Rev. Lett. 92, 073602 (2004).

[20] K. Baumann, C. Guerlin, F. Brennecke and T. Esslinger, Nature 464, 1301 (2010).

[21] K. Baumann, R. Mottl, F. Brennecke, T. Esslinger, arXiv:1105.0426v1 (2011).

[22] O. Castaños, E. Nahmad-Achar, R. López-Peña and J. G. Hirsch, Phys. Rev. A 83, 051601 (2011).

[23] O. Castaños, E. Nahmad-Achar, R. López-Peña, and J. G. Hirsch, On the superradiant phase in field-matter interactions, arXiv:1105.1153v1.

[24] T. Holstein and H. Primakoff, Phys. Rev. 58, 1098 (1940).

[25] J.M. Radcliffe, J. Phys. A 4, 313 (1971).

[26] A. Perelomov, Generalized Coherent States and Their Applications, Springer-Verlag (1986). 